\title{
Spiritual Care durch Seelsorge
}

Zum Beitrag der evangelischen Kirche im Gesundheitswesen

Eine Handreichung der

( Ständigen Konferenz für Seelsorge in der EKD

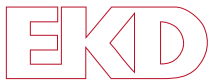

Evangelische Kirche in Deutschland 



\section{Spiritual Care durch Seelsorge}

Zum Beitrag der evangelischen Kirche im Gesundheitswesen

Eine Handreichung der

Ständigen Konferenz für Seelsorge in der EKD 


\section{Herausgeber}

Evangelische Kirche in Deutschland (EKD)

Herrenhäuser Str. 12 | 30419 Hannover

www.ekd.de

November 2020

Bestellung: versand@ekd.de

Download: www.ekd.de/publikationen

Satz: Büro Schroeder, Hannover

klimaneutral auf 100\% Recyclingpapier gedruckt

\section{INFO SERVICE \\ Evangelische Kirche \\ (i) $0800-5040602$ \\ =■info@ekd.de}




\section{Inhalt}

Hinführung

1. Wurzeln der Spiritual Care

2.Seelsorgliche Spiritual Care:

Integration und Fremdperspektive im Krankenhaus

3. Partikularistische und generalistische Kompetenz seelsorglicher Spiritual Care

4. Seelsorgliche Spiritual Care als Ander-Ort und Ander-Zeit

5. Spiritual Care und Ethik

6. Aus- und Fortbildung: Qualifikationskurse „Spiritual Care in Palliative Care“

7. Finanzierungsmodelle

8. Fazit

9. Empfehlungen 



\section{Hinführung}

Krankenhausseelsorge ist Kirche - für Patientinnen und Patienten, Angehörige sowie Mitarbeiterinnen und Mitarbeiter. Der Bedarf an Seelsorge in Krankenhäusern nimmt zu; dies ließ sich bereits vor dem Ausbruch der Corona-Pandemie feststellen. In Zeiten abnehmender kirchlicher Ressourcen stellt sich die Frage, wie lange die klassische Krankenhausseelsorge noch kirchlich umfänglich finanziert werden kann. Zugleich kommen Impulse aus der Palliativmedizin, sich auch von Krankenhausseite nicht nur auf Körperbehandlung und -pflege, sondern auch auf die geistigen und seelischen Bedürfnisse von Schwerstkranken und Sterbenden zu konzentrieren. Unter dem Leitbegriff Spiritual Care wird danach gefragt, wie sich die unterschiedlichen Berufsgruppen im Krankenhaus interprofessionell um den kranken Menschen und seine nicht nur leiblichen und psychischen, sondern auch spirituellen Bedürfnisse kümmern können und wie Seelsorge dabei integriert werden kann. Dabei ist nur bedingt eine professionelle Rollenaufteilung vorgesehen - alle sollen an Spiritual Care beteiligt werden - auch Ärzt*innen, Pflegepersonal und andere therapeutische Berufsgruppen. Wie dabei das Verhältnis von Seelsorge und Spiritual Care näher zu bestimmen ist, wird gegenwärtig ebenso diskutiert wie die Frage, ob an dieser Stelle ein kirchliches Seelsorgeangebot in den Refinanzierungsmechanismus des allgemeinen Gesundheitswesens einbezogen werden könnte. 


\section{Wurzeln der Spiritual Care}

Die Initiative für Spiritual Care hat ihren Ursprung in der Medizin, insbesondere der Palliativmedizin. Cicely Saunders, Krankenschwester und Ärztin in England, gilt als Begründerin der Hospizbewegung und der Palliative Care. Sie bezog Spiritual Care auf eine Spiritual Pain, die Sterbende durchleiden. Diese bedarf nach Saunders ebenso der Fürsorge und Aufmerksamkeit wie körperliche Schmerzen bzw. psychisches Leiden. Saunders orientierte sich dabei ganz selbstverständlich an christlichen Narrativen, nicht zuletzt an der Aufforderung des leidenden Jesus an seine Jünger in Gethsemane: „Wachet mit mir“ (Mt 26,38b). Saunders folgert daraus für die Sterbebegleitung: „We are not there to take away or explain, or even to understand but simply to ,Watch with me“". (vergleiche Cicely Saunders: Spiritual Pain, in: Cicely Saunders. Selected writings 1958-2004, hg. von David Clark, Oxford 2009, 217-221, 219) Sie unterstreicht, dass es in der Situation des Sterbens nicht zuerst darum geht, Sinn und Bejahung des Sterbeprozesses anzustreben, sondern geduldig die „spiritual pain“ des bzw. der Sterbenden mit ihm zusammen zu ertragen. Spiritual Care sollte deshalb nicht mit SinnSorge gleichgesetzt werden. Sie ist primär eine solidarische Begleitung kranker und sterbender Menschen; sie hält Verzweiflung aus und zwingt Menschen nicht in eine positive Interpretation schweren Leidens hinein. Als Seelsorge begleitet sie Menschen im Horizont des Evangeliums. 


\section{Seelsorgliche Spiritual Care: Integration und Fremdperspektive im Krankenhaus}

Spiritual Care versteht sich als ein ganzheitliches Behandlungskonzept, das die spirituelle Bedürfnisdimension kranker Menschen systematisch einschließt und mitberücksichtigt und damit die Lebensqualität von Schwerstkranken verbessern möchte. Prinzipiell können sich alle im Krankenhaus Tätigen an Spiritual Care beteiligen. Es ist deshalb sinnvoll, eine seelsorgliche und eine gesundheitsberufliche Spiritual Care zu unterscheiden. (vergleiche Simon Peng-Keller Spiritual Care: Grundgestalten, Leitmodelle und Entwicklungsperspektiven Spiritual Care: Basic forms, guiding models and perspectives for development https://doi.org/10.1515/spircare-2019-0078) In den Gesundheitsberufen ist Spiritual Care ein Teilaspekt professionellen Handelns und des achtsamen Umgangs mit Patient*innen und ihren Angehörigen, während in der Seelsorge ganz allgemein und damit auch für eine seelsorgliche Spiritual Care der spirituell-religiöse Aspekt die Kerndimension professionellen Handelns bzw. des persönlichen Gespräches bildet.

Seelsorger ${ }^{\star}$ innen sind unter dem Dach von Spiritual Care struktureller Teil eines multiprofessionellen Behandlungsteams. Sie kooperieren eng mit den anderen Berufsgruppen im Krankenhaus. Die Vorteile dieser Integration liegen in einer größeren Reichweite und besseren Einbindung seelsorglicher Arbeit, insbesondere im Hinblick auf den Ruf nach Seelsorge in der Sterbesituation. Auch sind Seelsorger*innen selbstverständlich Mitglieder in Ethikberatungsteams. Der Nachteil ist, dass die Unabhängigkeit von Seelsorger*innen dadurch eingeschränkt ist. Sie müssen sich mit Ärzt*innen absprechen, an Teambesprechungen teilnehmen und werden durch die spirituelle Anamnese und die daraus resultierenden Vorschläge für „spirituelle Interventionen“ in ihrer Handlungsfreiheit beeinträchtigt.

Das Changieren zwischen Integration in den klinischen Alltag und religiöser Fremdperspektive ist kennzeichnend für die seelsorgliche Spiritual Care. Seelsorge ist im Kontext von Spiritual Care dabei einerseits als Bestandteil der vierten Säule des Gesundheitswesens zu verstehen, zugleich steht Seelsorge mit ihrer religiösen Perspektive in Differenz zur dominierenden Perspektive des Gesundheitssystems. Deutlich wird dies beispielsweise bei ihrer Deutung des Sterbeprozesses, der nicht notwendig auf Akzeptanz und Versöhnung zielt, sondern bereit ist, gegebenenfalls auch das Sinnlose eines unbegreiflichen Sterbens im Sinne des „Wachet mit mir“ zu ertragen, um es 
gerade so dem betroffenen Menschen zu ermöglichen, auf die Art und Weise zu sterben, die er oder sie selbst für angemessen hält, auch wenn der oder die Sterbende dadurch für das Betreuungspersonal „unbequem" werden sollte oder sich weigert, seine ihm klinisch zugewiesene "Sterberolle“ einzunehmen. (vergleiche Irmhild Saake, Armin Nassehi, Katharina Mayr 2019: Gegenwarten von Sterbenden. Eine Kritik des Paradigmas vom »bewussten« Sterben, in: Kölner Zeitschrift für Psychologie und Sozialpsychologie 71, S. 27-52)

Prinzipiell stellt sich die Frage, wie Seelsorge und Spiritual Care so aufeinander bezogen werden können, dass Seelsorge einerseits Teil der interprofessionellen Spiritual Care ist und andererseits zugleich ihre eigene, spezifisch christliche Wahrnehmung zum Ausdruck bringt. Mit anderen Worten: Wie und worin treffen sich Seelsorge und Spiritual Care mit ihren je eigenen Systemlogiken ohne im Ergebnis zu unklaren Zuschreibungen und Erwartungen zu kommen. 


\section{Partikularistische und generalistische Kompetenz seelsorglicher Spiritual Care}

Seelsorge wird durch Spiritual Care gleichzeitig auf- und abgewertet. Sie wird aufgewertet, weil sie von Krankenhausseite nicht mehr als verzichtbar eingeschätzt, sondern Teil des Beziehungssystems der Patient ${ }^{\star}$ innen ist. Sie wird abgewertet, insofern Seelsorge im Vergleich zu einem spirituell allumfassenden Spiritual Care Giver auf eine konkrete Religion bezogen ist und damit nicht so flexibel eingesetzt werden kann wie eine vermeintlich weltanschaulich neutrale Spiritual Care.

Gesondert müssen in diesem Bereich kirchliche oder diakonische Häuser betrachtet werden. Denn bei allem Finanzdruck und bei aller Fachlichkeit setzen diese Häuser das Ziel um, in ihrer Arbeit auch ihren christlichen Auftrag und ihre christliche Prägung sowohl wirksam als auch sichtbar werden zu lassen. Seelsorge wird in der Diakonie deshalb als wichtiger und integraler Bestandteil der Gesamtausrichtung eines diakonischen Krankenhauses verstanden - auch angesichts aller Schwierigkeiten, die das im DRG-Betrieb mit sich bringen kann.

Aus Sicht des weithin säkularen Gesundheitssystems scheint der neutrale Spiritual Care Giver die naheliegendste und praktikabelste Lösung zu sein. Dabei ist allerdings zu bedenken, dass auch der weltanschaulich neutrale Spiritual Care Giver durch bestimmte Grundannahmen über den Menschen, Gott und die Welt geprägt ist. Seine Neutralität ist insofern nur eine vermeintliche. Christliche Seelsorge bezieht sich wiederum auf eine bestimmte Tradition und ist durch ihre Kenntlichkeit und Konkretheit erstens transparent (und damit womöglich weniger manipulativ) und vermag dadurch zweitens eigene Ressourcen und Resonanzräume für die seelsorgliche Begegnung zu erschließen. Konfessionell-religiös geprägte Seelsorgeangebote, die auf Glaubensgemeinschaften verweisen und an diese zurückgebunden sind, können demnach nicht leichthin durch eine generalistische spirituelle Begleitung ersetzt werden.

Die meisten Menschen im Krankenhaus sind im Lauf ihres Lebens mit bestimmten Formen von Religion und Spiritualität in Kontakt gekommen. Sie wollen in dieser ihrer je eigenen Religiosität bzw. Spiritualität wahrgenommen und auch angesprochen werden und hoffen beim Spiritual Care Giver auf entsprechende religiöse Deutungs- und Ritualangebote. Seelsorger ${ }^{\star}$ innen werden dabei nicht nur als religiöse Expert*innen, sondern auch als Personen, die selbst religiös sind, wahr- und als Menschen in An- 
spruch genommen, die authentisch etwas über ihren Glauben sagen können. Professionelle Seelsorge lebt deshalb davon, dass Seelsorger ${ }^{\star} i n n e n$ auch in einem über ihre konkrete Rolle hinausgehenden, persönlichen, transprofessionellen Sinn präsent sind.

Zugleich sind Seelsorger*innen nicht nur partikularistisch für die eigenen Glaubensgenoss*innen zuständig und ansprechbar, sondern auch Vertrauenspersonen für konfessionslose oder andersreligiöse Menschen, die über Spiritualität, Religion oder auch ganz andere Themen reden möchten. Seelsorger ${ }^{\star}$ innen verbinden insofern ihre partikularistische mit einer generalistischen Kompetenz in Sachen Spiritualität. Deshalb ist - wenn dies den Erwartungen der Personen, die auf Spiritual Care hoffen, entspricht - auch eine interreligiös gestaltete Seelsorge möglich und zu fördern. 


\section{Seelsorgliche Spiritual Care als Ander-Ort und Ander-Zeit}

Traugott Roser weist im Anschluss an Michel Foucault auf das heterochrone und heterotope Potential der Seelsorge im Krankenhaus und damit auf die vielfältigen Ressourcen einer spezifisch christlichen Seelsorge hin - in Form von Räumen der Stille und Klinikkapellen als Ander-Orte und in Form von Bibelworten, Gebeten, Segenshandlungen und Riten als Erfahrung von Ander-Zeiten. Ander-Orte und Ander-Zeiten stellen Gegenwelten dar. Sie ermutigen durch ihre Andersheit zur konstruktiv-kritischen Wahrnehmung dessen, was am „Normalort“ (in der „Normalzeit“) geschieht. Diese Andersheit ist der Ankerpunkt, von dem aus der Gefahr der Instrumentalisierung und Funktionalisierung der Religion durch das Medizinsystem zu wehren ist. Seelsorge ist religiöse Kommunikation und nicht medizinische Behandlung, sie orientiert sich an der Leitdifferenz transzendent/immanent (vergleiche Isolde Karle, Lehrwerk der Praktischen Theologie, Leipzig 2020, 403ff und 442ff) und nimmt zugleich aus dieser Perspektive ganzheitlich sensibel wahr, wie sich körperliches und psychisches Leiden auf Seele und Geist auswirkt. Das gilt auch für die spezifische Rolle der Seelsorge als seelsorgliche Spiritual Care. Sie kann im Krankenhaus insofern ein Ander-Ort und eine Ander-Zeit sein, die den Krankenhausalltag unterbricht und ein besonderes Potential erschließt.

So haben die Seelsorger*innen durch ihre Sonderstellung außerhalb krankenhaustypischer Routinen Zeit, wie sie keine andere Berufsgruppe im Krankenhaus in dieser Weise hat. Sie stehen außerhalb der ökonomischen Zwänge der Organisation. Die Seelsorgenden können es sich überdies leisten, auf ein steuerndes Handeln, auf das die Ärzt*innen beispielsweise verpflichtet sind, zu verzichten. Sie können akzeptieren, dass Patient*innen womöglich gar nicht über ihr Sterben reden und ihre Sterberolle nicht einnehmen möchten. Die Seelsorger*innen vermögen sich ganz auf die Gegenwart zu konzentrieren und lassen sich von den Impulsen, die von den Patient*innen kommen, leiten. Sie erweisen sich gerade darin als religiös, dass sie mit mehr Unbestimmtheit umgehen können, als es jeder anderen Kommunikations- und Interaktionsform möglich ist. (vergleiche Irmhild Saake, Armin Nassehi, Katharina Mayr 2019: Gegenwarten von Sterbenden. Eine Kritik des Paradigmas vom „bewussten“ Sterben, in: Kölner Zeitschrift für Psychologie und Sozialpsychologie 71, S. 27-52) 


\section{Spiritual Care und Ethik}

Die seelsorgliche Spiritual Care hat es vor allem im Krankenhaus, aber auch im Hospiz und im Altenpflegeheim mit schwerwiegenden ethischen Fragen zu tun. Es ist deshalb zu begrüßen und zu fördern, dass Seelsorger*innen im Rahmen von Spiritual Care in ethische Beratungsteams und Ethik-Komitees im Krankenhaus eingebunden sind und bei Konfliktsituationen an den Konsultationen teilnehmen.

Die ethische Dimension von Seelsorge steht seit einiger Zeit verstärkt im Fokus. Es ist deshalb elementar, dass Seelsorger*innen nicht nur über seelsorglich-pastoralpsychologische Kompetenzen und Weiterbildungen verfügen, sondern sich auch ethisch gut informiert mit komplexen Grenzfragen auseinandergesetzt haben und eine entsprechende Expertise besitzen. Seelsorger ${ }^{\star}$ innen sollten ein ethisch differenziertes Urteilsvermögen entwickeln, das ihnen hilft, auf die existentiellen Fragen, die ihnen gestellt werden, reflektiert, behutsam und gegebenenfalls auch beratend einzugehen. Seelsorgliche Spiritual Care ist als ethische Praxis zu gestalten, in der kranke, verletzliche Menschen im Zentrum der Aufmerksamkeit stehen. 


\section{Aus- und Fortbildung: Qualifikationskurse „Spiritual Care in Palliative Care“}

Kirchliche Seelsorger*innen, die einerseits mit anderen Berufsgruppen im Krankenhaus, in Hospizen und Pflegeeinrichtungen kooperieren und andererseits das Andere der eigenen Tradition transparent zur Geltung bringen, bedürfen nachweisbarer Kompetenzen.

Die Ausbildung zur Krankenhausseelsorge erfolgt für Pfarrer*innen und Diakon*innen in der Regel durch ihre mehrjährige akademische und praktische Ausbildung, die um pastoralpsychologische Weiterbildung wie eine Klinische Seelsorge-Ausbildung ergänzt wird. Spiritual Care im Sinne interprofessioneller Zusammenarbeit verlangt neben theologischen (hermeneutischen, ethischen und rituellen) und pastoralpsychologischen Kompetenzen auch kontextuale Kompetenzen, die spezifisch auf das Praxisfeld vorbereiten, in dem die Seelsorger*innen tätig sind. Im Bereich der Palliativmedizin, in dem gegenwärtig die Konzipierung von Spiritual Care am weitesten vorangeschritten ist, hat sich zu diesem Zweck ein Qualifizierungskurs Palliative Care etabliert, der gezielt kirchliche Akteur*innen auf die Seelsorge in palliativen und hospizlichen Einrichtungen vorbereitet. In Aufbau und Inhalt entspricht er den Qualifizierungskursen der anderen Gesundheitsberufe und umfasst in der Regel 120 Stunden. Die Kurse werden an spezialisierten Fort- und Weiterbildungseinrichtungen angeboten, sind gemäß den Richtlinien der DGP (Deutsche Gesellschaft für Palliativmedizin) zertifiziert und werden multiprofessionell unterrichtet. Spezifische Krankheitsbilder, Belastungen, Bedürfnisse sowie Arbeitsformen aller beteiligten Berufsgruppen, ethische Fragestellungen und Schnittstellen zur Seelsorge gehören unter anderem zur Ausbildung, ebenso die Beteiligung von Seelsorger*innen in Pflegeschulen und Medizinstudium.

Die Herausforderungen eines hochqualifizierten Umfelds machen es erforderlich, dass Seelsorger*innen sich über entsprechende kontextbezogene Bildungsangebote laufend weiterbilden. In palliativmedizinischen und hospizlichen Einrichtungen gilt der Nachweis der Teilnahme an einem Qualifizierungskurs als Voraussetzung für Spiritual Care. Es ist deshalb sinnvoll, entsprechende Standards zu entwickeln.

Auch die Qualifikation Ehrenamtlicher sollte für die Begleitung Schwerkranker und Sterbender ausgebaut werden. Die entsprechenden Qualifikationskurse und Beauf- 
tragungsstrukturen in den Gliedkirchen setzen hierfür einen strukturellen und inhaltlichen Rahmen, der durch feldspezifische Module erweitert und in Kooperation mit Seelsorger*innen aus verschiedenen Feldern (Klinik- und Altenheimseelsorge, Gemeinde etc.) sowie den Hospizdiensten, in denen bereits ehrenamtliche Mitarbeiter*innen als Spiritual Care Givers im Bereich der Spezialisierten Ambulanten Palliativversorgung (SAPV) tätig sind, durchgeführt werden kann.

Da Spiritual Care auch einen Teilaspekt des professionellen Handelns der Gesundheitsberufe darstellt, bedarf es einer Qualitätssicherung auch bei der gesundheitsberuflichen Spiritual Care durch entsprechende Bildungsmaßnahmen. Die DGP hat entsprechende Grundsätze zur Qualifizierung nichtseelsorglicher Berufsgruppen im Bereich Spiritual Care formuliert. Die Grundsätze umfassen Inhalte zur Arbeit von und mit Seelsorge ebenso wie Einheiten zum Verständnis von Krankheit und Sterben in unterschiedlichen Religionen. Zentral für alle Qualifizierungsmaßnahmen ist die Klärung der Begriffe Spiritualität, Religiosität, Glaube sowie die Entwicklung einer respektvollen Haltung als Basis jeder Form der Zusammenarbeit. Gerade um ein generalistisches, neutrales Verständnis von Spiritual Care zu vermeiden, bedarf es in den Qualifizierungskursen der nichtseelsorglichen Berufsgruppen einer Klärung des eigenen Verständnisses und der eigenen Spiritualität. Für eine Spiritual Care, die kirchliche Seelsorge als integralen Teil versteht, ist es geboten, Bildungsmaßnahmen und Qualifizierungskurse mit Seelsorger*innen gemeinsam und ökumenisch zu entwickeln und durchzuführen. Wo möglich, sollten auch Vertreter*innen anderer Religionen mit eingebunden sein, insofern diese über eigene Erfahrungen und Konzepte zu Seelsorge und Spiritual Care verfügen. 


\section{Finanzierungsmodelle}

Seelsorge ist bis heute selbstverständliches und unentgeltliches Angebot der Kirchen, das nach dem Grundgesetz in Krankenhäusern und anderen Einrichtungen offeriert werden darf, um die Religionsausübung gerade auch dort zu ermöglichen. Die Basisfinanzierung tragen die Religionsgemeinschaften.

Spiritual Care akzentuiert demgegenüber spirituelle Begleitung unter der Voraussetzung, dass Spiritual Pain sich auch gesundheitlich auswirkt und spirituelle Begleitung eine gesundheitsfördernde Wirkung hat. Damit gehörten prinzipiell alle Maßnahmen von der Befragung von Patient ${ }^{\star}$ innen, ob sie Seelsorge in Anspruch nehmen möchten, über die spirituelle Anamnese bis hin zu konkreten seelsorglichen Angeboten zum Bereich der Gesundheitsversorgung und wären von den Kostenträgern dann entsprechend zu gewährleisten. In der Palliativmedizin ist seelsorgliche Begleitung als Spiritual Care in die stationäre palliative Komplexbehandlung integriert und kann bei den Kostenträgern entsprechend geltend gemacht werden. Die Inanspruchnahme von Seelsorge stellt dieser Argumentation folgend sowohl den Ausdruck des Rechts auf freie Religionsausübung als auch auf eine bestmögliche gesundheitliche Versorgung dar. Seelsorge entwickelt unter der Perspektive einer public health insofern auch ein ausgeprägtes (gesundheits)politisches Profil.

Neben den bisher in Deutschland bestehenden Modellen der Finanzierung, Teilfinanzierung und Refinanzierung von Seelsorgestellen durch die Kirchen und die jeweiligen Krankenhausträger eröffnet das Konzept von Spiritual Care einen dritten Weg zur Refinanzierung durch das Gesundheitswesen. Damit verbunden sind allerdings zahlreiche zu klärende Fragen, die aus der prinzipiellen Andersheit von Seelsorge resultieren. Es ist der vollständigen Instrumentalisierung und Funktionalisierung von Seelsorge durch die Medizin zu wehren und damit letztlich der unverletzlichen Würde der Patient ${ }^{\star}$ innen zu dienen. Zu den problematischen Fragen gehört, wie die heilsamen Wirkungen von Seelsorge professionsspezifisch beschrieben werden und zugleich der auf Evidenzen basierenden Wirkungslogik des Gesundheitswesens entsprechen können. Ebenso ist die Frage ungeklärt, wie die Dokumentationspflicht sämtlicher Dienstleistungen in Gesundheitsberufen mit dem Seelsorgegeheimnis und der seelsorgerlichen Verschwiegenheit zu vereinbaren ist. Nicht zu unterschätzen ist schließlich die Frage, ob mit einer Finanzierung durch das Gesundheitswesen Seelsorge zu einer 
Verfügungsmasse der Gesundheitspolitik wird. Für eine Klärung dieser Fragen wäre es zu empfehlen, von den Erfahrungen in den Nachbarländern zu lernen, etwa dem Vereinigten Königreich, in dem die Krankenhausseelsorge durch das National Health System finanziert wird. 


\section{Fazit}

In der Sorge um kranke und sterbende Menschen haben viele verschiedene medizinische, religiöse und spirituelle Perspektiven und Kompetenzen ihren Ort. Die interprofessionelle Sorge um Kranke lässt sich in der Vielfalt der Maßnahmen und Perspektiven konstruktiv aufeinander beziehen, ohne die Differenz der Perspektiven dabei zu leugnen. Damit dies gelingt, sollte sich die Klinikseelsorge aktiv und kreativ an der Ausgestaltung einer interprofessionellen Spiritual Care und den damit verbundenen Lernprozessen beteiligen. Spiritual Care spielt dabei zunehmend auch jenseits des Krankenhauses eine wichtige Rolle (im Hospiz, aber auch in anderen care communities in ambulanten Versorgungsstrukturen, bei denen der gemeindlichen Seelsorge eine wichtige Funktion zukommen kann).

Spiritual Care steht von medizinischer Seite unter der Erwartung, der zunehmenden religiös-spirituellen Pluralität gerecht zu werden. Das ist eine zentrale Herausforderung für die seelsorgliche Spiritual Care, die dem Entkonfessionalisierungsdruck nicht nachgeben, den spezifisch religiösen Anliegen und Bedürfnissen von Schwerkranken und Sterbenden Rechnung tragen, in ihrem eigenen Profil erkennbar und zugleich inter- und transkonfessionell tätig sein sollte. Es geht darum - und das bereits akademisch durch die Praktische Theologie -, eine erkennbare Selbstverortung in der eigenen Tradition mit einer inter- und transreligiösen Offenheit zu verknüpfen. Eine christliche Spiritual Care kann ein zentrales kirchliches Engagement im Gesundheitswesen sein und ist als solches zu fördern - auch finanziell. Das verlangt freilich nach einer weitergeführten komplexen Verhältnisbestimmung, die sich im Kern um die Frage einer Kopplung der gesellschaftlichen Funktionssysteme Religion und Gesundheit dreht. So versteht sich diese Übersicht als Diskussionsbeitrag zu Aspekten der Gegenwartsrelevanz christlicher Religion sowie ihrer Eigenständigkeit und der Möglichkeit, Seelsorge in andere gesellschaftliche Felder mit den diesen eigenen Logiken und Dynamiken einzubeziehen. 


\section{Empfehlungen}

(1) Krankenhausseelsorge bleibt eine elementare kirchliche Aufgabe. Sie stellt Neuund Erstkontakte mit Kirche her und orientiert sich zugleich an ihren Mitgliedern. Spiritual Care bietet die Chance, die Seelsorge in einem zentralen kirchlichen Bereich, der Begleitung Pflegedürftiger, Schwerkranker und Sterbender, stationär und ambulant strukturell zu verankern. Sie öffnet damit die Möglichkeit, über den Bestand der bisherigen Krankenhausseelsorgestellen hinaus, im Rahmen weiterer Refinanzierungsmodelle Stellen oder Stellenanteile auszubauen bzw. zu stabilisieren. Die Basis hierfür bildet das Können der Seelsorger*innen und die lange Erfahrung mit dieser Arbeit in Kliniken und anderen Einrichtungen des Gesundheitsbetriebs.

(2) Die Attraktivität des Einbezugs von Seelsorge in Spiritual Care ergibt sich in einer doppelten Hinsicht: Zum einen wird der Mehrwert von Seelsorge im Gesundheitswesen in einem zentralen Bereich wahrgenommen. Dadurch erhält Seelsorge im Krankenhausbetrieb einen anderen Status. Zudem rückt die Sterbebegleitung als Aufgabe der Gemeindeseelsorge und in der Spezialisierten Ambulanten Palliativversorgung (SAPV) neu in den Blick. Schließlich wäre die Kirche durch ihre Integriertheit ins Gesundheitswesen noch einmal wirksame Partnerin in der Diskussion sowohl über ethische Fragestellungen als auch über spirituelle Bedürfnisse von Patientinnen und Patienten im Gesundheitsbetrieb.

(3) Grund zum Widerspruch ist dort geboten, wo christliche Seelsorge durch eine generalistische Spiritual Care ersetzt werden soll. Seelsorgliche Spiritual Care ist auf die durch das Evangelium gewährte Freiheit angewiesen, derer sie sich verdankt.

(4) Viele schwerkranke Patient*innen sterben zu Hause oder im Pflegeheim. Parallel empfiehlt es sich daher, zukunftsfähige Konzepte für die Seelsorge in Pflegeeinrichtungen und in der SAPV zu entwickeln.

(5) Um Spiritual Care in diesem Sinn zu fördern, empfiehlt die SKS, die noch ausstehende Refinanzierung von kirchlicher Seelsorge im Rahmen von Spiritual Care in der stationären palliativmedizinischen Komplexbehandlung sowie der SAPV bei gleichzeitiger kirchlicher Entsendung anzustreben und entsprechende Modelle dafür zu entwickeln. 


\section{Die Ständige Konferenz für Seelsorge in der EKD}

\section{SKS}

Die 2010 ins Leben gerufene Konferenz arbeitet zu Themen in der Seelsorge, die von gesamtkirchlichem Interesse sind. Besonders wichtig sind Bedingungen der Seelsorge und Qualitätsstandards sowie ihre theologischen und interdisziplinären Dimensionen.

Die in die SKS berufenen Mitglieder stammen aus ganz unterschiedlichen Dienst- und Arbeitszusammenhängen.

In der Zeit der Entstehung dieser Schrift gehörten der SKS an:

OKR Dr. Frank Ahlmann, Kiel

Pastor Dr. Ingo Habenicht, Bielefeld

Prof. Dr. Isolde Karle, Bochum

KRin Sabine Kast-Streib, Karlsruhe

Prof. Dr. Kerstin Lammer, Hamburg

Prof. Dr. Traugott Roser, Münster

KR Jürgen Sohn, Düsseldorf

Pastor Matthias Steinleitner, Dortmund

Pfarrerin Verena Übler, München

OKR Dr. Christoph Vogel, Berlin (Vorsitzender)

OKR Andreas Jensen (Geschäftsführung) 


www.ekd.de 